CLINICAL STUDY

\title{
Expression of the CD11c gene in subcutaneous adipose tissue is associated with cytokine level and insulin resistance in women with polycystic ovary syndrome
}

\author{
Tao Tao ${ }^{1, *}$, Shengxian $\mathrm{Li}^{1, *}$, Aimin Zhao ${ }^{2}$, Yanyun Zhang ${ }^{3}$ and Wei Liu ${ }^{1}$ \\ ${ }^{1}$ Division of Endocrinology and Metabolism, Department of Internal Medicine and ${ }^{2}$ Division of Reproductive Endocrinology and Infertility, Department of \\ Obstetrics and Gynecology, Shanghai Jiaotong University School of Medicine, Renji Hospital, 1630 Dongfang Road, Pudong, Shanghai 200127, People's \\ Republic of China and ${ }^{3}$ Joint Immunology Laboratory of Institute of Health Sciences and Shanghai Institute of Immunology, Shanghai Institutes for \\ Biological Sciences, Chinese Academy of Sciences and Shanghai Jiao Tong University School of Medicine, Shanghai, People's Republic of China \\ (Correspondence should be addressed to W Liu; Email: sue_liuwei@163.com; Y Zhang; Email: yyzhang@sibs.ac.cn)
}

*(T Tao and S Li contributed equally to this work)

\begin{abstract}
Objective: Alterations in the phenotypes of macrophages in adipose tissue play a key role in inflammation and insulin resistance (IR). The phenotypes of macrophages in subcutaneous adipose tissue (SAT) and the relationship between proinflammation markers and IR in women with polycystic ovary syndrome (PCOS) remain unclear. The objectives of this study are to characterize the gene expression of macrophage markers and cytokines in the SAT of PCOS women and to estimate their relationships with circulating levels of cytokines and IR.

Methods: The cross-sectional study involves 16 PCOS women and 18 normal control women. Cytokines and macrophage markers in the circulation and SAT were determined using ELISA, quantitative PCR, or immunofluorescence staining. IR was estimated using the homeostasis model assessment (HOMA-IR).

Results: The gene expression levels of CD11c along with TNF $\alpha$ and leptin in SAT remained significantly higher in PCOS women than in normal women $(P<0.05)$. However, no significant differences were found in CD68 mRNA expression in SAT between women with and without PCOS $(P>0.05)$. Furthermore, CD11c mRNA abundance provided a stronger contribution to models predicting serum levels of $\mathrm{TNF} \alpha(\mathrm{sTNF} \alpha)$ than did CD68 mRNA abundance. Lastly, increased sTNF $\alpha$ was associated with increased HOMA-IR in PCOS women, and this association was independent of both overall and visceral adiposity. Conclusion: The high expression level of CD11c mRNA in SAT was proved to be an important feature in PCOS women. Furthermore, $C D 11 \mathrm{c}$ mRNA abundance made a stronger contribution to models predicting STNF $\alpha$ in which existing proinflammatory properties might significantly contribute to the pathogenesis of IR in PCOS women.
\end{abstract}

European Journal of Endocrinology 167 705-713

\section{Introduction}

Polycystic ovary syndrome (PCOS) is known to affect $6-10 \%$ of reproductive-aged women (1). Insulin resistance (IR) has been considered to be the most important etiological aspect of reproductive and metabolic abnormalities in PCOS, in part due to the high prevalence of obesity in this disorder $(2,3)$. A chronic, low-grade inflammation occurring in adipose tissue is at least in part responsible for obesity-induced IR (4). Recent evidence of subclinical inflammation is present in women with $\operatorname{PCOS}(5,6)$ and might play an important role in the pathogenesis of PCOS (7). However, the precise role of dominant cell-extrinsic mechanisms, such as alterations in the levels of circulating adipokines and the occurrence of metabolic tissue inflammation that modulate peripheral insulin action in women with PCOS, has not been established.

In recent years, the relationship between the inflammation status in adipose tissue and IR has attracted wide attention. Findings from recent studies indicate that adipose tissue inflammation and the related metabolic dysregulation are connected to the presence of several immune cell types that were infiltrating into the adipose tissue. These include not only macrophages but also lymphocytes and other cells $(8,9)$. Among the immune cells that infiltrate adipose tissue in obesity, macrophages are functionally and numerically dominant $(10,11)$. However, findings of Arkan et al. (12) showed that lack of the proinflammatory enzyme inhibitor of nuclear factor kappa-B kinase subunit $\beta$ (IKK $\beta)$ in macrophages does not 
develop in diet-induced IR. Interestingly, recent studies $(10,11)$ have revealed that classically activated (M1) macrophages, which release cytokines such as IL1 $\beta$, IL6, and TNF $\alpha$ creating a proinflammatory environment that blocks adipocyte insulin action, have a crucial role in contributing to the pathogenesis of dietinduced IR. Furthermore, temporal analysis of adipose tissue in obesity has revealed that the recruitment of M1 macrophages coincides with the appearance of necrotic adipocytes and with the onset of IR (13).

CD11c is a member of the $\beta 2$ integrins, which has been consistently reported to be unregulated in macrophages infiltrating white adipose tissue (14). Further, CD11c + macrophages are seen to encircle necrotic adipocytes $(15,16)$, thereby forming crownlike structures (13) and remodeling adipose tissue structures. Recently, a study showed that CD11c promoter-dependent conditional deletion of adipose tissue macrophages (ATMs) improves insulin sensitivity, which indicates that CD11c + subsets of human ATMs are much more important in pathophysiology of IR than previously thought (17). Furthermore, the findings of Wu et al. (18) suggest that CD11c deficiency in dietinduced obese mice does not alter weight gain but decreases inflammation and ameliorates IR and glucose intolerance. It is suggested that the conditions of CD1 $1 \mathrm{c}$ expression may deter obesity progression by inducing inflammation and IR in AT. CD11c + macrophages play a key role in obesity-induced IR and obesity is a common phenotype in women with PCOS. Therefore, an important consideration is whether CD11c+ macrophages, acting as a characteristic of proinflammatory subset of classically activated macrophages, are also implicated in the pathogenesis of IR in women with PCOS.

Despite the importance of CD11c expression in dietinduced obesity as a regular aspect of IR and inflammation, much less is known about CD11c expression in subcutaneous adipose tissue (SAT) and their systemic effect on IR in women with PCOS. Additionally, the mechanisms for adipose dysfunction and subclinical inflammation in the pathophysiology of IR in women with PCOS remain unclear. Thus, we performed this study to test the characterization of adipose tissue dysfunction and proinflammatory markers in the circulation and in SAT of women with PCOS in relation to IR.

\section{Materials and methods}

A total of 34 subjects were included in this study. They were recruited from the outpatient Department of Endocrinology and Gynecology of Shanghai Renji Hospital between 2009 and 2010. Of these patients (age range 18-35 years), there were 16 women with PCOS (BMI range $23.8-29.64 \mathrm{~kg} / \mathrm{m}^{2}$ ) and 18 control women (BMI range $25.23-28.19 \mathrm{~kg} / \mathrm{m}^{2}$ ). All patients with PCOS were recruited from among patients seeking care for oligomenorrhea and/or hirsutism at the outpatient wards of the participating clinics. The PCOS diagnosis was based on the National Institutes of Health 1990 criteria. These include: i) clinical evidence of hyperandrogenism and/or hyperandrogenemia; ii) oligo-ovulation; and iii) the exclusion of related disorders, including nonclassical 21-hydroxylasedeficient adrenal hyperplasia, hyperprolactinemia, thyroid dysfunction, Cushing's syndrome, or androgen-producing tumors (19). The control women with regular menstrual cycles and without a family history of endocrine abnormality or hirsutism were recruited through an advertisement in a local newspaper. Control subjects were enrolled into the study if they met the following eligibility criteria: i) a healthy state, as determined by medical history, physical examination, screening laboratory evaluations, and transvaginal ultrasound; ii) without known heredity for type 2 diabetes mellitus and hypertension; iii) absence of changes $(\geq 10 \%)$ in body weight during the last year preceding the screening; iv) normal exercise and drinking habits; and v) no current regular medication (such as oral contraceptives or other drugs known to alter inflammation status). All study evaluations and procedures were conducted in accordance with the guidelines of the Helsinki Declaration on human experimentation. The study was approved by the ethics committee of Shanghai Renji hospital and all subjects provided written informed consent.

\section{Laboratory analysis}

All laboratory evaluations were performed at $0800 \mathrm{~h}$ after an overnight fast during the early follicular phase (days 2-5) of a spontaneous menstrual cycle, except in subjects with amenorrhea $>3$ months who were examined randomly. Fasting glucose and insulin samples were stored at $4{ }^{\circ} \mathrm{C}$ and analyzed on the day of sampling. Plasma glucose was determined using the glucose oxidase methodology. All measurements were performed with Roche reagents (D 2400 and E170 Modular Analytics modules with Roche/Hitachi analyzers; Roche Diagnostics). Insulin levels were measured by RIA. The intra-assay coefficient of variation $(\mathrm{CV})$ of insulin assays was 5.5 and $<10 \%$ respectively. Insulin sensitivity was evaluated by the quantitative insulin sensitivity check index (QUICKI) method. QUICKI $=1 /(\log (\mathrm{I} 0) \quad(\mu \mathrm{U} / \mathrm{ml})+\log (\mathrm{GO})$ $(\mathrm{mg} / \mathrm{dl}))$. The IR index (IRI) was calculated using the homeostatic assessment model of IR (HOMA-IR). HOMA-IR $=($ FINS $\times$ FPG) $/ 22.5$ (FINS, fasting serum insulin; FPG, fasting plasma glucose). Islet $\beta$ cell function was evaluated by the homeostasis model assessment of $\beta$-cell function (HOMA- $\beta$ ), HOMA- $\beta=$ $20 \times \mathrm{FINS} /(\mathrm{FPG}-3.5)$. Competitive electrochemiluminescence immunoassays on the Elecsys autoanalyzer 2010 (Roche Diagnostics) were used to quantify serum 
total testosterone and estradiol levels. Sex hormonebinding globulin (SHBG) levels were measured by chemiluminescent immunoassay (Elecsys autoanalyzer 2010, Roche Diagnostics) and were validated for plasma SHBG (20). The CV for SHBG using this methodology was $6 \%$. Free testosterone values were calculated based on total testosterone and SHBG levels according to the method outlined by Vermeulen et al. (21) assuming an albumin concentration of $4 \mathrm{~g} / \mathrm{dl}$ (http://www.Issam.ch/freetesto.htm). Serum leptin (s-leptin), adiponectin, and TNF $\alpha$ were measured using a commercial ELISA kit (R\&D, Minneapolis, MN, USA). The intra-assay and interassay $\mathrm{CV}$ were below 6 and $10 \%$ respectively.

\section{Adipose tissue collection}

Adipose tissue samples $(\sim 500 \mathrm{mg})$ were obtained surgically from SAT through a small incision in the lower abdomen. Specimens were transported immediately to the laboratory in a HEPES salts buffer containing 4\% BSA and $2 \mathrm{mM}$ pyruvate $(\mathrm{pH} 7.4)$ and were finely minced. The tissue specimens were immediately frozen in liquid nitrogen and stored at $-80{ }^{\circ} \mathrm{C}$ until total RNA isolation was undertaken.

\section{RNA isolation, RT, and real-time quantitative PCR}

Total RNA was isolated from the SAT and cells using TRIzol regent (Invitrogen) and was purified using the Qiagen RNeasy Mini Kit (Qiagen). Isolated RNA was reverse transcribed into cDNA by following the protocol from Superscript III First-Strand Synthesis System (Invitrogen). The mRNA expression of leptin, adiponectin, TNF $\alpha, I L 6, I L 10$, peroxisome proliferator-activated receptor $\gamma(P P A R \gamma(P P A R G)), C D 11 c$, and CD68 was determined by real-time quantitative PCR (QPCR). The QPCRs were performed in triplicate using a LightCycler instrument (Roche Diagnostics) with SYBR Premix Ex Taq (TaKaRa, Dalian, Japan) and were normalized using $\beta$-actin. Assay gene expression primers used are indicated in Table 1. Oligonucleotide primers were designed using the Primer Express 2.0 software (ABI, New York, NY, USA). The primer was synthesized by Shanghai Sangon Biological Engineering Technology and Services Co., Ltd. (Sangon, Shanghai, China) Amplification was performed in $10 \mu \mathrm{l}$ reactions using an amount of $4 \mathrm{ng}$ mRNA equivalent and a two-step standard protocol was employed following the manufacturer's instructions: amplification was performed during 40 cycles (pre-denaturation: $10 \mathrm{~s}$, $95{ }^{\circ} \mathrm{C}$, one cycle; PCR: $5 \mathrm{~s}, 94{ }^{\circ} \mathrm{C}$; $31 \mathrm{~s}, 60^{\circ} \mathrm{C}, 40$ cycles; dissociation stage). All primers used were validated for PCR efficiency. For each sample, $\Delta C p$ values were determined ( $\mathrm{Cp}$ of the target gene minus $\mathrm{Cp}$ of the $\beta$-actin gene). Fold changes in expression were calculated according to the transformation: fold increase $=2^{- \text {difference in } \Delta \mathrm{Cp}}$.

\section{Immunofluorescence staining}

A small amount of tissue was fixed in 4\% PBS-buffered paraformaldehyde for immunofluorescence. Frozen $20 \mu \mathrm{m}$ sections cut from adipose tissue embedded in optimal cutting temperature compound (OCT) were fixed in acetone. Primary antibodies used were rabbit MABs to CD11c (Abcam, Cambridge, UK) for classically activated macrophages. For single-antibody stains, sections were treated with 10\% goat serum (Boster, Wuhan, China) and then washed in PBS before overnight incubation with primary antibody. Slides were then labeled with Cy3-conjugated rabbit antirat IgG (Jackson Laboratories, East Brisbane, QLD, Australia). Then slides were mounted with Dapi mocent media (Vector Labs, Inc., Burlingame, CA, USA) and examined under immunofluorescence microscopy (Nikon, Tokyo, Japan). Immunopositive CD11c+ expression cells were counted in a field of $0.04 \mathrm{~mm}^{2}$, and only those with nuclei observable by DAPI staining were counted.

\section{Statistical analysis}

Distributions of continuous variables were tested for normality by use of the Kolmogorov-Smirnov test. Portions of results not normally distributed, based on the normal quartile plot, were log-transformed for all statistical analyses and reported back-transformed in their original units. All results were reported as

Table 1 Primer sequences.

\begin{tabular}{lll}
\hline Gene & Forward $\left(5^{\prime}-3^{\prime}\right)$ & Reverse $\left(5^{\prime}-3^{\prime}\right)$ \\
\hline Leptin & ATTTCACACACGCAGTCAGTCTC & TCACGTTTCTGGAAGGCATAC \\
Adiponectin & GAGATCCAGGTCTTATTGTCC & CAATCCCACACTGAATGCTG \\
$T N F \alpha$ & GAGTGACAAGCCTGTAGCCC & GATGAGGTACAGGCCCTCTG \\
$C D 11 c$ & TGACATTGCATCGAAGCCCTC & TCCGTACCCTCAATGGCAAAG \\
$C D 68$ & ATGGCGGTGGAGTACAATGTGT & AGAATGATGCTCGAGTTGCTGC \\
$P P A R \gamma$ & CAGGCTTGCTGAACGTGAAG & GGAGCACCTTGGCGAACA \\
IL6 & GGTACATCCTCGACGGCATCT & GT GCCTCTTTGCTGCTTTCAC \\
IL10 & GGGAGAACCTGAAGACCCTCA & TGCTCTT GTTTTCACAGGGAAG \\
$\beta$-Actin & CCTTCTACAATGAGCTGCGTG & ACAGCCTGGATAGCAACGTAC \\
\hline
\end{tabular}


means, or geometric means for log-transformed variables, with 95\% CIs. Comparisons between groups were made using Student's $t$-test. $P$ values $<0.05$ were considered significant. All statistical analyses were performed using SPSS version 13.0 (Statistical Package for the Social Sciences).

With this assumption, the study had $>85 \%$ power to detect differences in adipose tissue mRNA expression between these two groups, given an $\alpha$-error of 0.05 . Gene expression differences were made using Wilcoxon and Mann-Whitney $U$ tests. The relationships between serum levels of cytokines and adipose tissue CD11c expression and IR were evaluated by Spearman's correlation tests. Stepwise multiple linear regression analysis was performed for selected data, with statistical significance defined as $P<0.05$. Two forward, stepwise, multiple linear regression analyses were performed. The first model was performed to determine the best model to predict IR as estimated by HOMA-IR considering biologically plausible predictors, i.e. age, BMI, WHR, adiponectin, leptin, and TNF $\alpha$. These independent variables were selected based on the evaluation of the literature and because their correlations with insulin sensitivity were significant in the univariate analyses. Based on the results of the first model, a second model was created to determine the best model to predict serum levels of TNF $\alpha$ considering age, BMI, WHR, and CD11c, CD11c/CD68 as plausible independent variables.

\section{Results}

\section{Hormonal and biochemical features}

The clinical characteristics and biochemical variables for the two groups of women according to PCOS status are summarized in Table 2. As expected, there were no significant differences in age, BMI, waist circumstances, WHR, fasting glucose, or estradiol between women with and without PCOS. However, mean total and free testosterone values were also significantly higher in the PCOS group compared with the BMI-matched control group $(P=0.003$ and $P=0.000$ respectively). Mean SHBG values were significantly lower in the PCOS group compared with the BMI-matched control group $(P=0.001)$. Meanwhile, fasting insulin and HOMA-IR values were significantly higher in women with PCOS than in the BMI-matched normal controls $(P=0.003$ and $P=0.011$ respectively). Mean HOMA-IS and QUICKI values were significantly lower in women with PCOS than in normal controls $(P=0.002$ and $P=0.013$ respectively).

\section{Characterization of adipose tissue dysfunction in PCOS patients}

To identify the features of adipose tissue dysfunction and subclinical inflammation state, we compared circulating level of adiponectin, leptin, and TNF $\alpha$. As shown in Table 2, serum levels of leptin and TNF $\alpha$ remained significantly higher in women with PCOS than in normal control women $(P=0.034$ and $P=0.000$ respectively). However, there were no significant differences in serum levels of adiponectin between women with and without PCOS $(P=0.157)$. Furthermore, we performed quantitative PCR to detect the relative amounts of mRNA for adiponectin, leptin, TNF $\alpha$, and PPAR $\gamma$ in SAT between women with and without PCOS. As shown in Fig. 1, the expression levels of leptin and TNF $\alpha$ mRNA in SAT were significantly higher in women with PCOS than those in BMI-matched controls $(P=0.039$ and $P=0.042$

Table 2 Clinical and biochemical variables in women with polycystic ovary syndrome (PCOS) and normal control women. Data are means $(95 \% \mathrm{Cl})$ unless noted.

\begin{tabular}{|c|c|c|c|}
\hline Variable & Control & Pcos & $P$ value \\
\hline$n$ & 18 & 16 & NA \\
\hline Age (years) & $25.4(24.3-26.6)$ & $24.6(22.1-27.2)$ & 0.517 \\
\hline $\mathrm{BMI}\left(\mathrm{kg} / \mathrm{m}^{2}\right)$ & $26.71(25.23-28.19)$ & $26.72(23.80-29.64)$ & 0.992 \\
\hline Waist circumference $(\mathrm{cm})$ & 87.19 (82.87-91.52) & $86.37(80.06-98.1)$ & 0.818 \\
\hline WHR & $0.88(0.85-0.91)$ & $0.87(0.84-0.90)$ & 0.54 \\
\hline $\mathrm{FBG}(\mathrm{mmol} / \mathrm{l})$ & $5.31(4.99-5.61)$ & $4.96(4.72-5.19)$ & 0.074 \\
\hline Fasting insulin (mIU/l) & $7.85(6.09-9.60)$ & $12.61(9.90-15.32)$ & 0.003 \\
\hline HOMA-IR & $1.84(1.42-2.28)$ & $2.79(2.17-3.40)$ & 0.011 \\
\hline HOMA-IS & $95.98(70.37-121.59)$ & $190.13(133.12-247.15)$ & 0.002 \\
\hline QUICKI & $0.36(0.34-0.37)$ & $0.33(0.32-0.35)$ & 0.013 \\
\hline Estradiol (pmol/l) & $295.17(197.02-393.32)$ & $225.07(150.35-299.77)$ & 0.238 \\
\hline Total testosterone $(\mathrm{nmol} / \mathrm{l})$ & $1.63(1.42-1.85)$ & $2.96(2.18-3.74)$ & 0.003 \\
\hline SHBG $(\mathrm{nmol} / \mathrm{l})$ & $62.71(51.12-74.29)$ & $36.23(27.68-44.77)$ & 0.001 \\
\hline Free testosterone ${ }^{*}(\mathrm{pmol} / \mathrm{l})$ & $19.75(16.44-23.06)$ & $53.74(38.85-68.62)$ & 0.000 \\
\hline Adiponectin (mg/l) & $9.47(7.99-10.94)$ & $7.88(6.07-9.70)$ & 0.157 \\
\hline Leptin $(\mu \mathrm{g} / \mathrm{l})$ & $41.08(29.14-52.93)$ & $61.97(45.45-78.13)$ & 0.034 \\
\hline $\mathrm{TNF} \alpha(\mathrm{ng} / \mathrm{l})$ & $30.69(22.41-38.98)$ & $68.66(49.52-87.80)$ & 0.000 \\
\hline
\end{tabular}

$P$ value for unpaired $t$-test for difference between two groups. WHR, waist-to-hip ratio; QUICKI, quantitative insulinsensitivity check index; HOMA-IR, homeostasis model assessment-insulin resistance index; FBG, fasting blood glucose; SHBG, sex hormone-binding globulin; to convert glucose to $\mathrm{mg} / \mathrm{dl}$, divide by $0.05551 .{ }^{*}$ geometric means. 

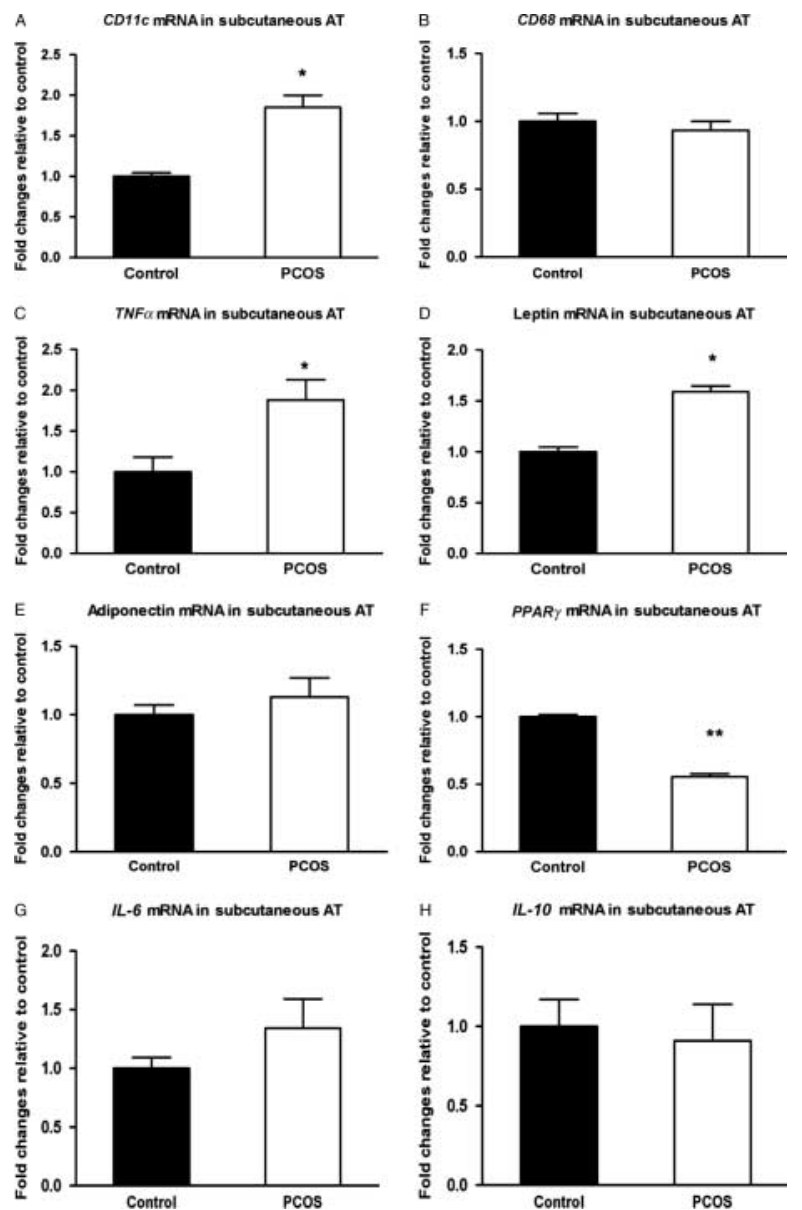

Figure 1 The gene expression of $C D 11 C(\mathrm{~A}), C D 68(\mathrm{~B}), T N F \alpha(\mathrm{C})$, leptin (D), adiponectin (E), PPAR $(\mathrm{F}), I L 6(\mathrm{G})$, and $I L 10(\mathrm{H})$ in adipose tissue in women with and without PCOS. Data are mean \pm S.E.M. $P$ value for Wilcoxon and Mann-Whitney $U$ tests for difference between two groups $<0.05$ for $C D 11 c, T N F \alpha$, leptin, adiponectin, $P P A R \gamma, I L 6$, and IL10. ${ }^{*} P<0.05,{ }^{* *} P<0.01$.

respectively). In addition, we found that the mRNA expression levels of adiponectin and ratios of leptin to adiponectin were similar between PCOS and non-PCOS groups ( $P=0.53$ and $P=0.077$ respectively). Lastly, the mRNA expression levels of PPAR $\gamma$ in SAT were significantly lower in women with PCOS than in BMI-matched controls $(P=0.008)$.

\section{Macrophage infiltration into adipose tissue and phenotype of ATM subsets}

In addition, we performed quantitative PCR to evaluate the mRNA expression levels of $C D 68$, a macrophagespecific marker, and CD11c in SAT between women with PCOS and controls. As shown in Fig. 1, the expression levels of $C D 11 c$ mRNA were significantly higher in women with PCOS than in BMI-matched controls $(P=0.036)$. However, the expression levels of CD68 mRNA and ratios of $C D 11 c$ to $C D 68$ mRNA were not significantly different between women with PCOS and normal control women $(P=0.677$ and $P=0.962$ respectively). Meanwhile, no significant differences in the mRNA expression levels of IL6 and IL1O were found between PCOS women and control subjects $(P=0.067$ and $P=0.14$ respectively).

To identify macrophage phenotypes present in adipose tissue, we performed immunofluorescent staining with specific antibodies. As shown in Fig. 2, the presence of macrophages, identified by positive staining for $\mathrm{CD} 11 \mathrm{c}$ in SAT, which is viewed as a marker of proinflammatory macrophages marker, clearly indicated the presence of specific subsets of macrophages in the SAT of women with PCOS.

\section{Correlation between CD11c mRNA expression levels and IR-related gene/biochemical characteristics}

As shown in Fig. 3, considering all women with PCOS, serum levels of both TNF $\alpha(r=0.824, P=0.000)$ and leptin $(r=0.582, \quad P=0.018)$ had positive linear relationships with the expression levels of CD11c

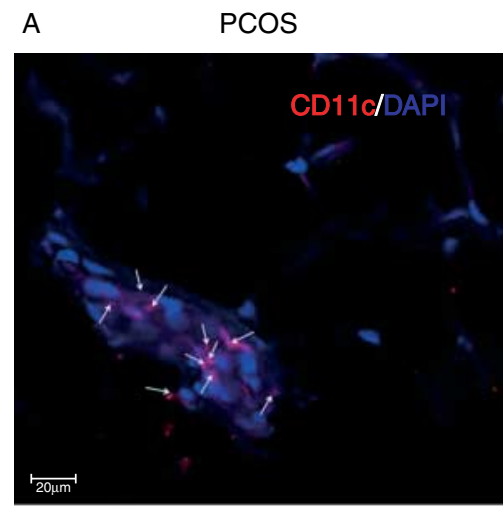

B Normal control

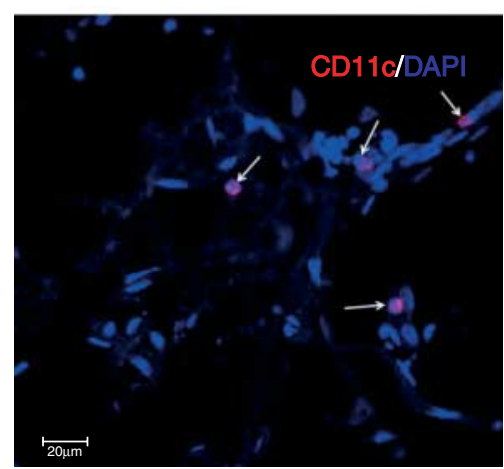

Figure 2 Immunofluorescence staining of CD11c-positive cells in SAT obtained from women with PCOS (A) and control women (B) (magnification, $\times 40$ ) is shown. The white arrow indicates CD11cpositive (red) cells in subcutaneous adipose tissue quantified by counting in a field of $0.04 \mathrm{~mm}^{2}$. 

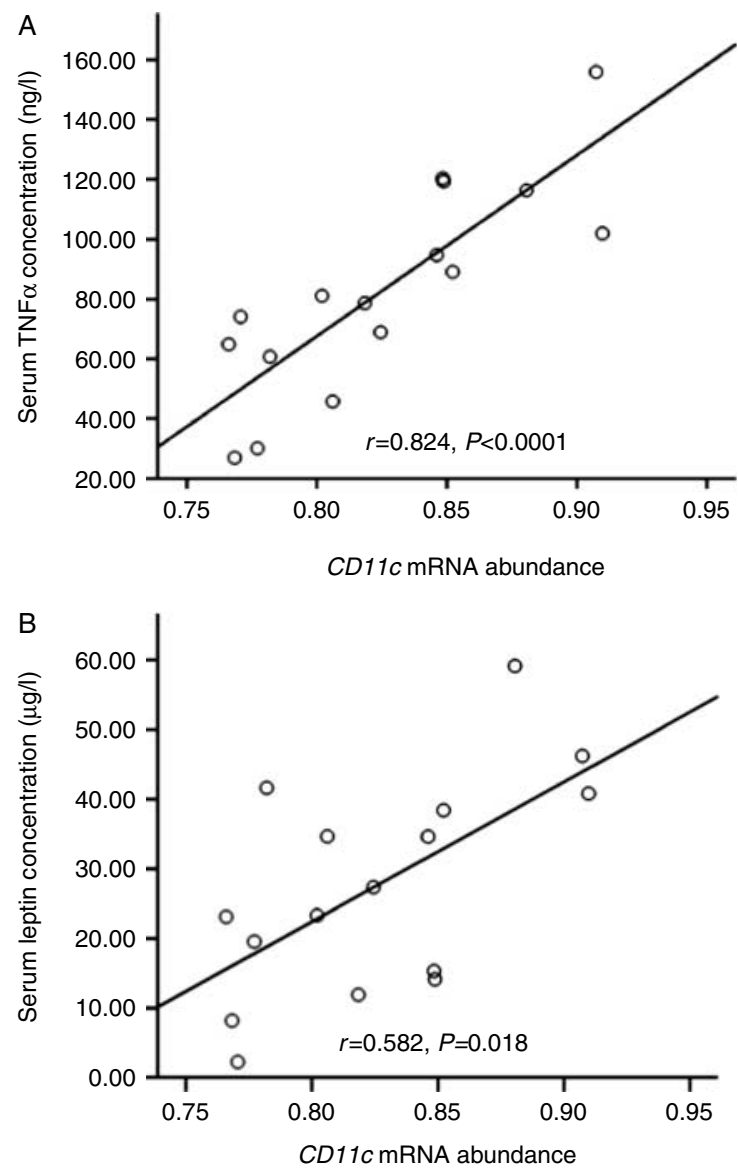

Figure 3 Relationship between $C D 11 c$ gene expression in adipose tissue and serum TNF $\alpha(A)$ and s-leptin (B) in women with PCOS $(n=16)$. $P$ value for Spearman's correlation tests.

mRNA. In addition, as shown in Fig. 4, a strong positive linear correlation was observed between CD11c mRNA expression levels and IR estimated by HOMA-IR $(r=0.577, \quad P=0.019)$. However, neither PPAR $\gamma$ mRNA expression $(r=-0.103, P=0.704)$ nor other gene (TNF $\alpha$, leptin, and adiponectin) expression levels correlated significantly with CD11c mRNA expression levels $(P>0.05$ for all). Finally, we do not find that CD11c and CD68 mRNA expression levels have strong linear relationships with estradiol or with total and free testosterone values $(P>0.05$ for all).

\section{Determinants of IR as estimated by HOMA-IR}

To investigate the independent determinants of IR among PCOS women studied, we performed a forward, stepwise, multiple regression analysis with HOMA-IR as the dependent variable and CD11c mRNA, BMI, s-leptin, and sTNF $\alpha$ as potential contributors to IR. In the analysis, only sTNF $\alpha$ was selected as an independent variable for the final model of HOMA-IR. In the final model for HOMA-IR, the adjusted $R^{2}$ was
$0.998(P<0.0001)$ and the regression coefficients $(\beta)$ were $0.001(P=0.003)$ for sTNF $\alpha$. Thus, sTNF $\alpha$ was an independent predictor of IR as estimated by HOMAIR in women with PCOS.

\section{Determinants of serum TNF $\alpha$}

As TNF $\alpha$ mainly secreted from classically activated macrophages, we hypothesized that CD11c mRNA abundance might be an important predictor of sTNF $\alpha$ level. After sTNF $\alpha$ was selected as a better predictor of HOMA-IR than BMI, CD11c mRNA, and s-leptin in the first linear regression analysis, a second model was created, again using forward, stepwise, linear regression analysis, with sTNF $\alpha$ as the dependent variable and CD11c mRNA, CD68 mRNA, and s-leptin as potential independent variables. Considering these variables, only CD11c mRNA was selected as an independent variable for the final model predicting sTNF $\alpha$. The adjusted $R^{2}$ for the model predicting sTNF $\alpha$ was $0.701(P=0.003)$, and the regression coefficients $(\beta)$ were $1.118(P=0.000)$. Thus, the expression level of CD11c mRNA was an independent predictor of sTNF $\alpha$ level in women with PCOS.

\section{Discussion}

In diet-induced obesity, most of the infiltrating ATMs were polarized into the M1 phenotype and the presence of a high number of these CD11c + macrophages was associated with a local inflammation status and IR in adipose tissue (22). However, data concerning the link among CD11c + macrophages, system inflammation, and IR in women with PCOS are limited. The issue of whether the change in inflammation in adipose tissue poses a major defect for IR in women with PCOS was also unsettled. The main finding of this study is the

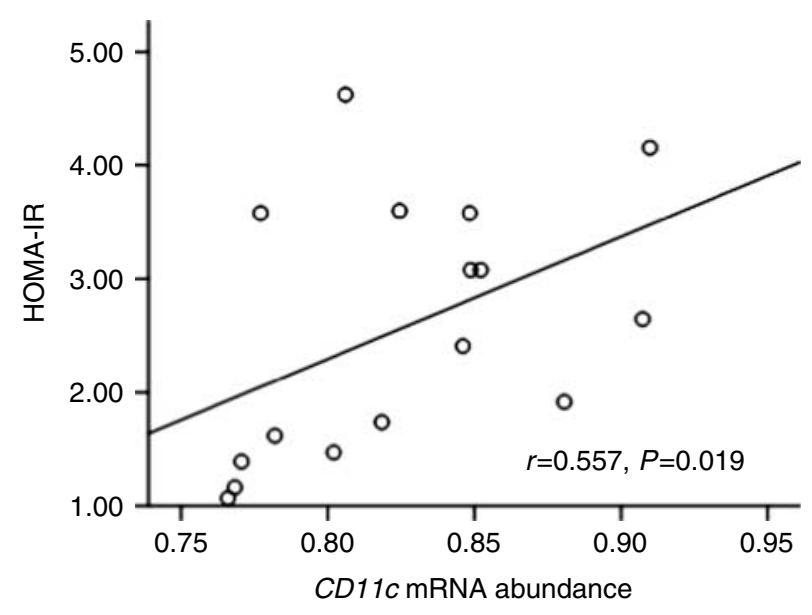

Figure 4 Relationship between $C D 11 c$ gene expression in adipose tissue and insulin resistance estimated by HOMA-IR in women with PCOS $(n=16)$. $P$ value for Spearman's correlation tests. 
demonstration that existing high expression levels of classically activated inflammatory macrophages in abdominal SAT, as estimated by CD11c expression, was an important feature found in women with PCOS. This kind of phenotypic switch of macrophage in adipose tissue is perhaps a more important indicator of IR than total macrophage infiltration as estimated by CD68 mRNA in adipose tissue in women with PCOS. In addition, the increased $C D 11 \mathrm{c}$ mRNA expression level is seen to be related to increased sTNF $\alpha$ and s-leptin levels in PCOS women. Furthermore, sTNF $\alpha$ provided a stronger contribution to models predicting IR than did leptin or adiponectin in women with PCOS. Lastly, CD11c mRNA provided a stronger contribution to models predicting sTNF $\alpha$ level in women with PCOS.

It is noteworthy that chronic low-level inflammation, a key contributor to the pathogenesis of PCOS, was shown in recent studies $(23,24)$. However, previous studies that assessed the relationship between subclinical inflammation and IR in women with PCOS have yielded conflicting results $(25,26)$. Based on our present data, we find that, compared with BMI-matched controls, serum TNF $\alpha$ level is significantly increased in women with PCOS. Additionally, in a forward stepwise, linear regression analysis performed to determine predictors of IR as estimated by HOMA-IR in women with PCOS, STNF $\alpha$ was shown to be an independent predictor of HOMA-IR after controlling for BMI and age. However, we found that neither s-leptin nor adiponectin were independent predictors of HOMA-IR after controlling for BMI in this study. Therefore, we speculated that TNF $\alpha$ may be more important than leptin and adiponectin in predicting IR in women with PCOS after controlling for BMI and may be involved in the development of PCOS. The observed association between TNF $\alpha$ and IR in our study is consistent with other studies suggesting that the TNF $\alpha$ has the most potent IR properties (27) in women with PCOS. Moreover, our results are in agreement with the previous studies showing that serum levels of inflammatory markers are increased in overweight patients with PCOS compared with weight-matched controls (28). However, in conflict with our results, Olszanecka-Glinianowicz et al. (29) reported that they failed to display any differences between overweight controls and overweight patients with PCOS in terms of circulating TNF $\alpha$ and IL6. In addition, our data show that the gene expression level of TNF $\alpha$ in SAT is obviously higher in women with PCOS than in BMImatched control women. However, we do not find that the expression levels of IL6 mRNA were significantly different between women with PCOS and normal control women. Findings from this study seem to contrast with the reports from the laboratories of Lindholm et al. (30). They showed that overweight rather than the PCOS diagnosis per se appears to be the main explanatory variable for elevated adipose tissue inflammation in patients with PCOS. Several explanations for the apparent disparity in these findings can be hypothesized, including differences in study subject demographics (specifically ethnicity, different PCOS criteria) and clinical characteristics (i.e. higher prevalence of obesity in this study). In our study, we used the 1990 NIH PCOS criteria, but the studies by Lindholm et al. (30) based their diagnosis of PCOS on the Rotterdam diagnostic criteria, and thus represent a more heterogenous group of women. Lastly, the correlation analyses do not show sTNF $\alpha$ correlated with the expression of TNF $\alpha$ mRNA in SAT in this study. This may suggest that alterations in sTNF $\alpha$ in women with PCOS contribute to the phenotypic presentation of the syndrome via additional mechanisms dependent on posttranscriptional regulation.

As TNF $\alpha$ mainly secreted from ATMs exists in close correlation with IR in women with PCOS, we speculate that macrophage infiltration into adipose tissue should act as a feature of paramount importance, leading to adipose-derived inflammation and IR in women with PCOS. We do not find that the expression levels of CD68, IL6, and IL10 mRNA in SAT have a significant difference between PCOS women and BMI-matched control women though. However, we find that increased CD11c mRNA abundance was associated with sTNF $\alpha$ $(P=0.000)$ and HOMA-IR $(P=0.019)$ in women with PCOS. More importantly, immunofluorescence staining of CD11c clearly indicates the presence of specific subsets of macrophages in the SAT in women with PCOS in this study. Hence, our results suggested that alterations in phenotype of ATMs may contribute to the system inflammation and IR in women with PCOS. However, the precise mechanism of macrophage activation remains unknown. Our data suggest that the PCOS diagnosis per se appears to be the main explanatory variable for elevated adipose tissue inflammation in women with PCOS. These findings seem to support the reports from the laboratories of González et al. They show that hyperandrogenism may be a potential mechanism for inflammation in PCOS by activating mononuclear cells (MNC) in the fasting state and increasing MNC sensitivity to glucose (31). Unfortunately, we do not find that $C D 11 c$ and $C D 68$ mRNA expression levels have strong linear relationships with serum estradiol or with total and free testosterone values. Interestingly, we find that the expression of enzymes synthesizing testosterone and enzymes inactivating DHT and progesterone are higher in SAT of PCOS patients compared with BMI-matched controls in our previous study (32). Moreover, correlation analysis indicated that increased leptin expression may be negatively related to local DHT level. This suggests that a complex network among local sex hormone and adipocytokines exits in SAT in women with PCOS. Furthermore, Alemany (33) suggested that the differences in androgen availability and handling may help to explain some of the sex-related differences in the onset and development of the metabolic syndrome. 
Hence, a possible explanation may lie in the role of the local sex hormones in macrophage phenotype switch through changing the microenvironment of adipocytes, which increases local immune response and inflammation in PCOS women. However, given the cross-sectional design of our study, causality cannot be established. The precise mechanisms of macrophage activation in SAT in women with PCOS must be further investigated.

Several limitations of our study should be considered. The study includes a limited number of participants, which is the reason we cannot separate subtle groups according to BMI, but we instead analyze the data after adjusting for BMI. Another limitation is that only adipose tissue biopsies from one anatomical location were available for this study. Recently, genomic and proteomic profiling studies of morbidly obese PCOS omental adipose tissue have revealed changes in components of several biological pathways $(34,35)$. Hence, there might be differences in the inflammatory status of visceral adipose tissue in women with PCOS (36). However, it should be emphasized that the subcutaneous fat in the truncal region appears to contribute more to both the IR and the circulating NEFA pool than do adipose tissue depots elsewhere in the body (37), and it is not likely that the visceral tissue would directly account for the increased circulating concentrations of plasma cytokines and chemokines $(38,39)$. Finally, CD11c + is not the only marker for staining M1-macrophages known in humans. However, $\mathrm{CD} 11 \mathrm{c}+$ macrophage acting as an important characteristic of pro-inflammation was associated with a local inflammation status in diet-induced obesity. On the other hand, we found decreased expression levels of PPAR $\gamma$ mRNA in SAT in women with PCOS in this study, which gene can stimulate M2 polarization of ATMs and change systemic insulin sensitivity (40).

In conclusion, we find an important link between macrophage activation status and IR in PCOS women in this study. The remodeling of SAT might be proposed to be active participants in the development of IR in women with PCOS. Further investigation is warranted to determine both the mechanisms by which inflamed adipose tissue is altered in PCOS and the potential contributions of alterations in the proinflammatory cytokines to ovulatory dysfunction in PCOS women.

\section{Declaration of interest}

The authors declare that there is no conflict of interest that could be perceived as prejudicing the impartiality of the research reported.

\section{Funding}

This work was supported by the Natural Science Foundation of Shanghai, China (12ZR1417800 to T Tao, 2012) and Shanghai Science and Technology Development Fund (08411953000 to T Tao, 2008).

\section{Acknowledgements}

The authors thank the research volunteers for their participation in this study. They also thank their study nurse Chen Ya-wen for excellent assistance with the clinical measurements and sample arrangements, and the staff of the department of gynaecology and obstetrics for their invaluable help with this project.

\section{References}

1 Diamanti-Kandarakis E, Kouli CR, Bergiele AT, Filandra FA, Tsianateli TC, Spina GG, Zapanti ED \& Bartzis MI. A survey of the polycystic ovary syndrome in the Greek island of Lesbos: hormonal and metabolic profile. Journal of Clinical Endocrinology and Metabolism 199984 4006-4011. (doi:10.1210/jc.84.11. 4006)

2 Dunaif A. Insulin resistance and the polycystic ovary syndrome: mechanism and implication for pathogenesis. Endocrine Reviews 199718 774-800. (doi:10.1210/er.18.6.774)

3 Hahn S, Tan S, Sack S, Kimmig R, Quadbeck B, Mann K \& Janssen OE. Prevalence of the metabolic syndrome in German women with polycystic ovary syndrome. Experimental and Clinical Endocrinology \& Diabetes 2007115 130-135. (doi:10.1055/ s-2007-967093)

4 Gregor MF \& Hotamisligil GS. Inflammatory mechanisms in obesity. Annual Review of Immunology 201129 415-445. (doi:10.1146/annurev-immunol-031210-101322)

5 Escobar-Morreale HF, Luque-Ramírez M \& González F. Circulating inflammatory markers in polycystic ovary syndrome: a systematic review and metaanalysis. Fertility and Sterility 2011 95 1048-1058.e1-2. (doi:10.1016/j.fertnstert.2010.11.036)

6 Samy N, Hashim M, Sayed M \& Said M. Clinical significance of inflammatory markers in polycystic ovary syndrome: their relationship to insulin resistance and body mass index. Disease Markers 200926 163-170. (doi:10.3233/DMA-2009-0627)

7 Repaci A, Gambineri A \& Pasquali R. The role of low-grade inflammation in the polycystic ovary syndrome. Molecular and Cellular Endocrinology 2011335 30-41. (doi:10.1016/j.mce. 2010.08.002)

8 Lolmède K, Duffaut C, Zakaroff-Girard A \& Bouloumié A. Immune cells in adipose tissue: key players in metabolic disorders. Diabetes \& Metabolism 201137 283-290. (doi:10.1016/j.diabet. 2011.03.002)

9 Lumeng CN \& Saltiel AR. Inflammatory links between obesity and metabolic disease. Journal of Clinical Investigation 2011121 2111-2117. (doi:10.1172/JCI57132)

10 Ferrante AW Jr. Obesity-induced inflammation: a metabolic dialogue in the language of inflammation. Journal of Internal Medicine 2007262 408-414. (doi:10.1111/j.1365-2796.2007. 01852.x)

11 Odegaard JI \& Chawla A. Alternative macrophage activation and metabolism. Annual Review of Pathology 2011 6 275-297. (doi:10.1146/annurev-pathol-011110-130138)

12 Arkan MC, Hevener AL, Greten FR, Maeda S, Li ZW, Long JM, Wynshaw-Boris A, Poli G, Olefsky J \& Karin M. IKK- $\beta$ links inflammation to obesity-induced insulin resistance. Nature Medicine 200511 191-198. (doi:10.1038/nm1185)

13 Strissel KJ, Stancheva Z, Miyoshi H, Perfield JW II, DeFuria J, Jick Z, Greenberg AS \& Obin MS. Adipocyte death, adipose tissue remodeling, and obesity complications. Diabetes 200756 2910-2918. (doi:10.2337/db07-0767)

$14 \mathrm{Wu} \mathrm{H}$, Gower RM, Wang H, Perrard XY, Ma R, Bullard DC, Burns AR, Paul A, Smith CW, Simon SI \& Ballantyne CM. Functional role of $\mathrm{CD} 11 \mathrm{c}+$ monocytes in atherogenesis associated with hypercholesterolemia. Circulation 2009119 2708-2717. (doi:10.1161/CIRCULATIONAHA.108.823740)

15 Cinti S, Mitchell G, Barbatelli G, Murano I, Ceresi E, Faloia E, Wang S, Fortier M, Greenberg AS \& Obin MS. Adipocyte death 
defines macrophage localization and function in adipose tissue of obese mice and humans. Journal of Lipid Research $2005 \mathbf{4 6}$ 2347-2355. (doi:10.1194/jlr.M500294-JLR200)

16 Lumeng CN, Bodzin JL \& Saltiel AR. Obesity induces a phenotypic switch in adipose tissue macrophage polarization. Journal of Clinical Investigation 2007117 175-184. (doi:10.1172/JCI29881)

17 Patsouris D, Li PP, Thapar D, Chapman J, Olefsky JM \& Neels JG. Ablation of CD11c-positive cells normalizes insulin sensitivity in obese insulin resistant animals. Cell Metabolism 20088 301-309. (doi:10.1016/j.cmet.2008.08.015)

18 Wu H, Perrard XD, Wang Q, Perrard JL, Polsani VR, Jones PH, Smith CW \& Ballantyne CM. CD11c expression in adipose tissue and blood and its role in diet-induced obesity. Arteriosclerosis, Thrombosis, and Vascular Biology 201030 186-192. (doi:10.1161/ATVBAHA. 109.198044)

19 Zawadski JK \& Dunaif A. Diagnostic criteria for polycysticovary syndrome: towards a rational approach. In Current Issues in Endocrinology and Metabolism: Polycystic Ovary Syndrome, pp 377-384. Eds A Dunaif, J Givens, F Haseltine \& G Merriam, New York: Blackwell, 1992.

20 Reynders M, Anckaert E, Schiettecatte J \& Smitz J. Evaluation of a new automated electrochemiluminescent sex hormone-binding globulin (SHBG) immunoassay. Clinical Chemistry and Laboratory Medicine 200543 86-89. (doi:10.1515/CCLM.2005.013)

21 Vermeulen A, Verdonck L \& Kaufman JM. A critical evaluation of simple methods for the estimation of free testosterone in serum. Journal of Clinical Endocrinology and Metabolism $1999 \mathbf{8 4}$ 3666-3672. (doi:10.1210/jc.84.10.3666)

22 Chawla A, Nguyen KD \& Goh YP. Macrophage-mediated inflammation in metabolic disease. Nature Reviews. Immunology 201111 738-749. (doi:10.1038/nri3071)

23 González F. Inflammation in polycystic ovary syndrome: underpinning of insulin resistance and ovarian dysfunction. Steroids 201277 300-305. (doi:10.1016/j.steroids.2011.12.003)

24 González F, Rote NS, Minium J \& Kirwan JP. Increased activation of nuclear factor kappaB triggers inflammation and insulin resistance in polycystic ovary syndrome. Journal of Clinical Endocrinology and Metabolism 2006 91 1508-1512. (doi:10.1210/jc.2005-2327)

25 Kelly CCJ, Lyall H, Petrie JR, Gould GW, Connell JMC \& Sattar N. Low grade chronic inflammation in women with polycystic ovarian syndrome. Journal of Clinical Endocrinology and Metabolism 200186 2453-2455. (doi:10.1210/jc.86.6.2453)

26 Mohlig M, Spranger J, Osterhoff M, Ristow M, Pfeiffer AF, Schill T, Schlösser HW, Brabant G \& Schöfl C. The polycystic ovary syndrome per se is not associated with increased chronic inflammation. European Journal of Endocrinology 2004150 525-532. (doi:10.1530/eje.0.1500525)

27 Hotamisligil GS, Shargill NS \& Spiegelman BM. Adipose expression of tumor necrosis factor- $\alpha$ : direct role in obesity-linked insulin resistance. Science 1993259 87-91. (doi:10.1126/science.7678183)

28 GonzalezF, Rote NS, Minium J \& Kirwan JP. Evidence of proatherogenic inflammation in polycystic ovary syndrome. Metabolism $2009 \mathbf{5 8}$ 954-962. (doi:10.1016/j.metabol.2009.02.022)

29 Olszanecka-Glinianowicz M, Banas M, Zahorska-Markiewicz B, Janowska J, Kocelak P, Madej P \& Klimek K. Is the polycystic ovary syndrome associated with chronic inflammation per se? European Journal of Obstetrics \& Gynecology and Reproductive Biology 2007 133 197-202. (doi:10.1016/j.ejogrb.2006.10.037)
30 Lindholm A, Blomquist C, Bixo M, Dahlbom I, Hansson T, Sundström Poromaa I \& Burén J. No difference in markers of adipose tissue inflammation between overweight women with polycystic ovary syndrome and weight-matched controls. Human Reproduction 201126 1478-1485. (doi:10.1093/humrep/der096)

31 González F, Nair KS, Daniels JK, Basal E \& Schimke JM. Hyperandrogenism sensitizes mononuclear cells to promote glucose-induced inflammation in lean reproductive-age women. American Journal of Physiology. Endocrinology and Metabolism 2012 302 E297-E306. (doi:10.1152/ajpendo.00416.2011)

32 Wang L, Li S, Zhao A, Tao T, Mao X, Zhang P \& Liu W. The expression of sex steroid synthesis and inactivation enzymes in subcutaneous adipose tissue of PCOS patients. Journal of Steroid Biochemistry and Molecular Biology 2012132 120-126. (doi:10.1016/j.jsbmb.2012.02.003)

33 Alemany M. Do the interactions between glucocorticoids and sex hormones regulate the development of the metabolic syndrome? Frontiers in Endocrinology 20123 27. (doi:10.3389/fendo.2012. 00027)

34 Corton M, Botella-Carretero JI, Benguria A, Villuendas G, Zaballos A \& San Millan JL. Escobar-Morreale HF, Peral B. Differential gene expression profile in omental adipose tissue in women with polycystic ovary syndrome. Journal of Clinical Endocrinology and Metabolism 200792 328-337. (doi:10.1210/ jc.2006-1665)

35 Corton M, Botella-Carretero JI, Lopez JA, Camafeita E, San Millan JL, Escobar-Morreale HF \& Peral B. Proteomic analysis of human omental adipose tissue in the polycystic ovary syndrome using two-dimensional difference gel electrophoresis and mass spectrometry. Human Reproduction 200823 651-661. (doi:10.1093/humrep/dem380)

36 Carmina E, Chu MC, Moran C, Tortoriello D, Vardhana P, Tena G, Preciado R \& Lobo R. Subcutaneous and omental fat expression of adiponectin and leptin in women with polycystic ovary syndrome. Fertility and Sterility $2008 \mathbf{8 9} 642-648$. (doi:10.1016/j.fertnstert. 2007.03.085)

37 Garg A. Regional adiposity and insulin resistance. Journal of Clinical Endocrinology and Metabolism $2004 \mathbf{8 9} 4206-4210$. (doi:10.1210/jc.2004-0631)

38 Murdolo G, Hammarstedt A, Sandqvist M, Schmelz M, Herder C, Smith U \& Jansson PA. Monocyte chemoattractant protein-1 in subcutaneous abdominal adipose tissue: characterization of interstitial concentration and regulation of gene expression by insulin. Journal of Clinical Endocrinology and Metabolism $2007 \mathbf{9 2}$ 2688-2695. (doi:10.1210/jc.2006-2814)

39 Despres JP \& Lemieux I. Abdominal obesity and metabolic syndrome. Nature $2006 \mathbf{4 4 4} 881-887$. (doi:10.1038/nature05488)

40 Odegaard JI, Ricardo-Gonzalez RR, Goforth MH, Morel CR, Subramanian V, Mukundan L, Red Eagle A, Vats D, Brombacher F, Ferrante AW \& Chawla A. Macrophage-specific PPARgamma controls alternative activation and improves insulin resistance. Nature 2007 447 1116-1120. (doi:10.1038/nature05894)

Received 21 April 2012

Revised version received 29 August 2012

Accepted 3 September 2012 